\title{
ROL DE LA TOMOGRAFÍA COMPUTADA DE ABDOMEN EN LA EVALUACIÓN DE GASTRECTOMÍA EN MANGA LAPAROSCÓPICA Y SUS COMPLICACIONES
}

\author{
Int. Cristóbal Teuber $\mathbf{S}^{(1)}$, Drs. Hugo Vidal $A^{(1)}$, Roberto Oyanedel $Q^{(2)}$, Andrés O'Brien $\mathbf{S}^{(3)}$.
}

1. Interno de Medicina. Pontificia Universidad Católica de Chile.

2. Departamento de Radiología. Hospital Clínico Pontificia Universidad Católica de Chile.

3. Departamento de Radiología. Clínica Las Condes. Chile.

\section{ROLE OF ABDOMINAL COMPUTED TOMOGRAPHY IN THE EVALUATION OF LAPAROSCOPIC SLEEVE GASTRECTOMY TECHNIQUE AND ITS COMPLICATIONS}

\begin{abstract}
Bariatric surgery has developed considerably over recent years due to the progressive increase in obesity worldwide. The laparoscopic sleeve gastrectomy is a new restrictive technique, with promising initial outcomes. CT images play an important role in postoperative evaluation as well as in complication management in patients undergoing this surgical technique. Here we review the role of abdominal computed tomography in the study of potential complications.
\end{abstract}

Keywords: Bariatric surgery, Complications, Obesity, Sleeve gastrectomy.

Resumen: La cirugía bariátrica se ha desarrollado considerablemente en el último tiempo como consecuencia del aumento progresivo de la obesidad a nivel mundial. La gastrectomía en manga laparoscópica es una técnica restrictiva nueva, con resultados iniciales prometedores. Las imágenes juegan un rol importante en la evaluación postoperatoria por lo que es imprescindible conocer los cambios anatómicos normales y las principales complicaciones de esta técnica. En este trabajo revisamos el rol de la tomografía computada de abdomen en el estudio de posibles complicaciones.

Palabras clave: Cirugía bariátrica, Complicaciones,

Teuber $\boldsymbol{C}$ y cols. Rol de la tomografía computada de abdomen en la evaluación de gastrectomía en manga laparoscópica y sus complicaciones. Rev Chil de Radiol 2010; 16(2): 70-72.

Correspondencia: Dr. Roberto Oyanedel $Q$. oyanedelroberto2@gmail.com, cjteubersanz@gmail.com Trabajo recibido el 25 de octubre de 2009, aceptado para publicación el 19 de abril de 2010.
Gastrectomía en manga, Obesidad.

\section{Introducción}

La obesidad es una enfermedad crónica y multifactorial, considerada la pandemia del presente siglo, debido al aumento progresivo de su prevalencia en prácticamente todos los países. En Chile, las cifras de sobrepeso y obesidad se acercan a las de Estados Unidos con un 33 y $31 \%$ de la población respectivamente ${ }^{(1)}$. En este contexto, la cirugía bariátrica se ha desarrollado considerablemente como una alternativa para los pacientes refractarios a tratamiento médico. Actualmente, el bypass gástrico y la banda gástrica ajustable son las técnicas más utilizadas ${ }^{(2)}$.

La gastrectomía en manga laparoscópica (GML) se ha transformado en una alternativa quirúrgica exitosa en el tratamiento de la obesidad. Se describió inicialmente como la primera etapa antes de cirugías más complejas como la derivación biliopancreática o el bypass gástrico ${ }^{(3)}$. Sin embargo, varios estudios han reportado resultados prometedores utilizando esta técnica como terapia única(4-7).

Los resultados de la GML en términos de reducción de peso a corto plazo, sumados a la baja tasa de complicaciones y la posibilidad de realizar una segunda intervención complementaria ante el fracaso terapéutico, hacen esperable un aumento progresivo en la utilización de esta técnica.

\section{Gastrectomía en manga laparoscópica}

La GML es un procedimiento puramente restrictivo en el que se reseca la mayor parte del cuerpo gástrico, reduciendo su capacidad en un $80 \%$ y conservando un pequeño tubo o manga gástrica a nivel de la curvatura menor. La técnica se realiza por vía laparoscópica e incluye la disección del ligamento gastrocólico en la curvatura mayor del estómago, coagulación de vasos gástricos cercanos al bazo, separación del cuerpo gástrico con ayuda de suturas mecánicas y finalmente extracción 
del segmento resecado en una bolsa ${ }^{(7)}$; algunos grupos incluyen además disección de un segmento del antro gástrico para evitar la distensión gástrica.

En relación a otras técnicas de cirugía bariátrica, las principales ventajas son: conserva la función gástrica, no requiere realizar una anastomosis digestiva ni intervenir el mesenterio, no se asocia a síndrome de dumping y permite realizar cirugías complementarias ante el fracaso terapéutico. Esto mantiene una adecuada absorción de nutrientes y medicamentos, disminuye la tasa de complicaciones (especialmente las relacionadas a la anastomosis y las hernias internas) e incluso, se asocia a una mejor calidad de vida ${ }^{(6)}$.

\section{Evaluación por tomografía computada}

El cambio anatómico de la GML consiste en un reservorio gástrico, asociado a la curvatura menor, con un píloro intacto. El reservorio es de mayor tamaño que el observado en el bypass gástrico y tiene forma tubular. Las suturas lineales pueden ser visualizadas en la tomografía computada como cuerpos hiperdensos.

Las imágenes tienen un rol fundamental en la evaluación de pacientes con sospecha de complicación y en el diagnóstico diferencial correspondiente ${ }^{(13)}$. Las complicaciones que se deben buscar dirigidamente en el abdomen son: presencia de colecciones, líquido libre intraperitoneal, neumoperitoneo, solución de continuidad de la línea de sutura, extravasación de contraste oral o i.v., dilatación de la manga gástrica y áreas de estenosis, trombosis venosa e infartos esplénicos.

Las tasas de complicaciones reportadas alcanzan un $10,3 \%$, considerándose mayores hasta en un 5,8\% ${ }^{(9)}$ de los casos. La complicación más frecuente reportada es la fístula gástrica postoperatoria (FGP) que puede representar hasta un $5,1 \%$ del total de complicaciones ${ }^{(7,9)}$. Con frecuencias variables en los distintos estudios se incluyen: estenosis de la gastroplastía, hemorragia de la línea de sutura y enfermedad tromboembólica $(T V P-T E P)^{(4,9)}$. Entre las complicaciones menores se encuentran atelectasias, insuficiencia renal transitoria, necesidad de soporte ventilatorio por más de 24 horas y vómitos. La tasa de re-hospitalización alcanza un $2,7 \%$ en los primeros tres meses ${ }^{(10)}$ y la mortalidad asociada es de $0,9 \%$, representada principalmente por FGP y hemorragias ${ }^{(9)}$.

\section{Fístula gástrica postoperatoria}

Como ya se mencionó, corresponde a la complicación más frecuente y principal responsable de la mortalidad asociada. La FGP suele presentarse durante la primera semana del post-operatorio, aunque se ha descrito una presentación a las 10 semanas ${ }^{(11)}$. Se debe fundamentalmente a fallas de la técnica y/o elección inadecuada del tamaño de las grapas de la sutura. Los síntomas iniciales son: taquicardia sin causa cardíaca, dolor abdomi- nal, disnea, confusión e hipotensión; dentro de las presentaciones graves destaca el shock séptico de origen intraabdominal, responsable del $50 \%$ de las muertes en cirugía bariátrica ${ }^{(12)}$. Cualquiera de estos síntomas, especialmente durante las primeras 72 horas, debe alertar al médico tratante que deberá solicitar exámenes de laboratorio e imaginológicos para descartar una FGP. Por otra parte, es importante descartar enfermedad tromboembólica que, si bien ocurre con menor frecuencia, se asocia a una mortalidad de hasta $30 \%{ }^{(12)}$.

Las alteraciones tomográficas presentes en la FGP son: derrame pleural izquierdo, líquido libre en cavidad peritoneal, colección intraabdominal y extravasación de medio de contraste oral (Figura 1). El sitio más frecuente de filtración es el ángulo de His, en la unión esófago-gástrica.

El tratamiento inicial de esta complicación dependerá de la sintomatología del paciente y del tamaño del defecto. Pacientes asintomáticos son manejados en forma conservadora, mientras que en pacientes sintomáticos se prefiere el manejo quirúrgico. En fístulas pequeñas se prefiere el tratamiento endoscópico y en fístulas de mayor tamaño se opta por el tratamiento quirúrgico.

\section{Infarto esplénico}

El infarto esplénico es una complicación rara de la gastrectomía en manga, con pocos casos reportados $^{(14)}$. Se ha descrito con mayor frecuencia como complicación de la cirugía de bypass gástrico ${ }^{(15)}$ pero hemos diagnosticado algunos casos en nuestra institución; probablemente se relaciona con la manipulación de los vasos esplénicos durante la disección del ligamento gastrocólico y también la coagulación de arterias gástricas cercanas al bazo.

La presentación clínica es muy variable. El síntoma más frecuente es el dolor abdominal en hipocondrio izquierdo, presente en un $70 \%$ de los casos; sin embargo, hasta un tercio de los pacientes permanecen asintomáticos. El diagnóstico tomográfico se realiza por la ausencia de realce de un segmento del bazo con uso de medio de contraste i.v. (Figura 2).

El manejo del infarto esplénico es conservador. El manejo quirúrgico está indicado frente a síntomas persistentes o la presencia de complicaciones: hemorragia, rotura y formación de abscesos o pseudoquistes.

\section{Conclusión}

La TC juega un rol importante en la evaluación y manejo de las complicaciones post-operatorias en la GML. Considerando el aumento progresivo de la cirugía bariátrica en el control de la obesidad refractaria a tratamiento médico, creemos necesario conocer los cambios anatómicos habituales y tener presente las principales complicaciones asociadas a la técnica quirúrgica. 

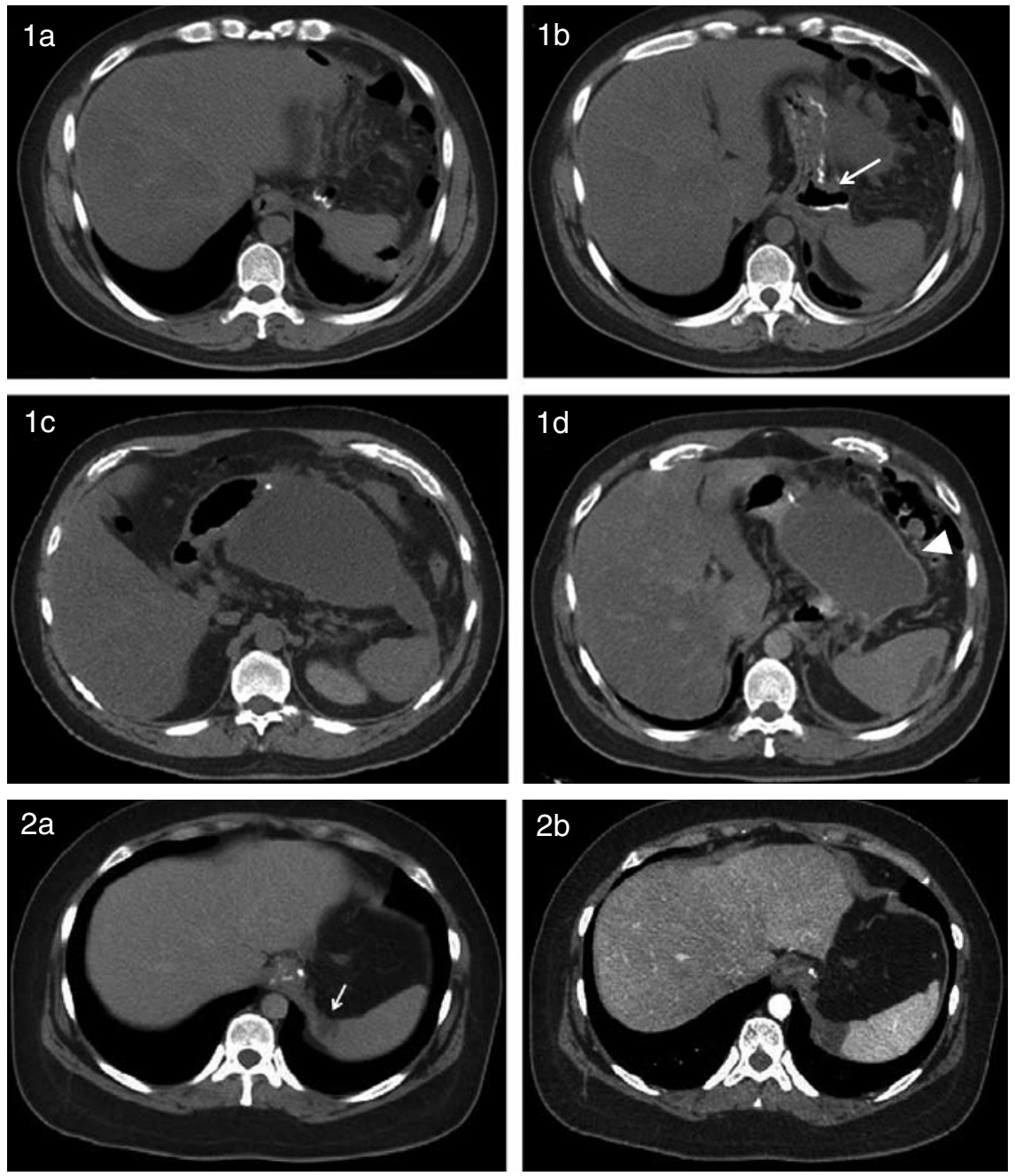

Figura 1. FGP diagnosticada por TC. Se observa una solución de continuidad de la sutura de gastrectomía en su aspecto proximal, asociada a extravasación de medio de contraste oral, flecha $(a, b)$ y colección de $15,7 \mathrm{~cm}$ de longitud (c) con pared gruesa que refuerza con medio de contraste, cabeza de flecha (d). No se demostró extravasación de medio de contraste intravenoso hacia la colección.

Figura 2. Infarto esplénico diagnosticado por TC. Se observa lesión esplénica hipodensa, flecha (a), sin impregnación con medio de contraste (b).

\section{Bibliografía}

1. Vio F. Prevención de la obesidad en Chile. Rev Chil Nutr 2005; 32(2): 80-7.

2. Buchwald $H$, Williams $S E$. Bariatric surgery worldwide 2003. Obes Surg 2004; 14: 1157-64.

3. Mognol P, Chosidow D, Marmuse JP. Laparoscopic sleeve gastrectomy as an initial bariatric operation for high-risk patients: initial results in 10 patients. Obes Surg 2005; 15: 1030-3.

4. Moon Han S, Kim WW, Oh JH. Results of laparoscopic sleeve gastrectomy (LSG) at 1 year in morbidly obese Korean patients. Obes Surg 2005; 15: 1469-75.

5. Roa P, Kaidar-Person O, Pinto D, Cho M, Szomstein S, Rosenthal R. Laparoscopic sleeve gastrectomy as treatment for morbid obesity: Technique and short-term outcome. Obes Surg 2006; 16(10): 1323-26.

6. Rubin M, Yehoshua RT, Stein M, Lederfein D, Fichman $\mathrm{S}$, Bernstine $\mathrm{H}$ et al. Laparoscopic sleeve gastrectomy with minimal morbidity. Early results in 120 morbidly obese patients. Obes Surg 2008; 18(12): 1567-70.

7. Fuks D, Verhaeghe P, Brehant O, Sabbagh C, Dumont F, Riboulot M, Delcenserie R, Regimbeau JM. Results of laparoscopic sleeve gastrectomy: A prospective study in 135 patients with morbid obesity. Surgery 2009; 145(1): 106-13.

8. Gumbs A, Gagner M, Dakin G, Pomp A. Sleeve gastrectomy for morbid obesity. Obes Surg 2007; 17: 962-9.

9. Iannelli A, Dainese R, Piche T, Facchiano E, Gugen- heim J. Laparoscopic sleeve gastrectomy for morbid obesity. World J Gastroenterol 2008; 14(6): 821-7.

10. Tucker O, Szomstein S, Rosenthal R. Indications for sleeve gastrectomy as a primary procedure for weight loss in the morbidly obese. J Gastrointest Surg 2008; 12: 662-7.

11. Montoya J, Olguin H, Aguilar O, Ceron M, Ortiz A. Reporte de un caso, paciente con antecedentes de gastrectomía tubular en manga que presento fístula gastrica a los 2.5 meses de postoperada. Presentado en XVIII Congreso Internacional de Cirugía Endoscópica 2009.

12. Barboza E, Barboza A, Calmet F, Montes M, Ronceros V, Málaga $G$ et al. Degastrectomía total salvadora de vida en sepsis abdominal post cirugía bariatrica de manga gástrica. Rev Gastroenterol Perú 2007; 27: 295-302.

13. Chandler RC, Srinivas G, Chintapalli KN, Schwesinger WH, Prasad SR. Imaging in bariatric surgery: A guide to postsurgical anatomy and common complications. AJR 2008; 190(1): 122-35.

14. Dhanasopon A, Lewis C, Folek J, Dutson E, Mehran A. Splenic infarct as complication of sleeve gastrectomy. Surg Obes Relat Dis 2009; 5(5): 626-629.

15. Yu J, Turner MA, Cho SR, Fulcher AS, DeMaria EJ, Kellum JM et al. Normal anatomy and complications after gastric bypass surgery: helical CT findings. Radiology 2004; 231(3): 753-60. 